\title{
The role of neuronavigation in intracranial endoscopic procedures
}

\author{
Veit Rohde • Timo Behm • Hans Ludwig • \\ Dorothee Wachter
}

Received: 8 January 2011 /Revised: 6 June 2011 / Accepted: 8 October 2011 /Published online: 16 December 2011

(C) The Author(s) 2011. This article is published with open access at Springerlink.com

\begin{abstract}
In occlusive hydrocephalus, cysts and some ventricular tumours, neuroendoscopy has replaced shunt operations and microsurgery. There is an ongoing discussion if neuronavigation should routinely accompany neuroendoscopy or if its use should be limited to selected cases. In this prospective clinical series, the role of neuronavigation during intracranial endoscopic procedures was investigated. In 126 consecutive endoscopic procedures (endoscopic third ventriculostomy, ETV, $n=65$; tumour biopsy/resection, $n=36$; non-tumourous cyst fenestration, $n=23$; abscess aspiration and hematoma removal, $n=1$ each), performed in 121 patients, neuronavigation was made available. After operation and videotape review, the surgeon had to categorize the role of neuronavigation: not beneficial; beneficial, but not essential; essential. Overall, neuronavigation was of value in more than $50 \%$ of the operations, but its value depended on the type of the procedure. Neuronavigation was beneficial, but not essential in 16 ETVs $(24.6 \%), 19$ tumour biopsies/ resections $(52.7 \%)$ and 14 cyst fenestrations (60.9\%). Neuronavigation was essential in 1 ETV (2\%), 11 tumour biopsies/ resections (30.6\%) and 8 cyst fenestrations (34.8\%). Neuronavigation was not needed/not used in 48 ETVs $(73.9 \%), 6$ endoscopic tumour operations (16.7\%) and 1 cyst fenestration (4.3\%). For ETV, neuronavigation mostly is not required. In
\end{abstract}

\footnotetext{
V. Rohde

Department of Neurosurgery, University of Aachen,

Aachen, Germany

V. Rohde $(\bowtie) \cdot$ T. Behm $\cdot$ H. Ludwig $\cdot$ D. Wachter

Department of Neurosurgery, Georg-August-University Göttingen,

Robert-Koch-Strasse 40,

37075 Göttingen, Germany

e-mail: veit.rohde@med.uni-goettingen.de
}

the majority of the remaining endoscopic procedures, however, neuronavigation is at least beneficial. This finding suggests integrating neuronavigation into the operative routine in endoscopic tumour operations and cyst fenestrations.

Keywords Neuroendoscopy · Neuronavigation · Third ventriculostomy

\section{Introduction}

Today, endoscopic third ventriculostomy (ETV) is the therapy of choice in the management of occlusive hydrocephalus $[4$, 5]. Arachnoidal and other intracranial cysts had been treated successfully by endoscopic cystoventriculostomy and cystocisternostomy [20,25]. For intra- and periventricular tumours, endoscopic techniques at least for biopsy are gaining increasing acceptance $[1,14,15]$. Most of these endoscopic operations are performed as free-hand procedures, with the risk of damage to vital neurovascular structures and the risk of loss of orientation, if clear anatomical landmarks are absent or obscured. Stimulated by reports on severe intraoperative complications in free-hand procedures [10, $19,21]$, endoscopy was combined with frame-based and, more recently, with frameless stereotaxy $[3,8,12,16]$. The question still is unanswered, if neuronavigation should be routinely made available during neuroendoscopic procedures to be prepared for unforeseen intraoperative difficulties, or if the decision for or against neuronavigation should be made individually on the base of preoperative imaging. The aim of this prospective study was to define the benefit of neuronavigation for successful completion of intracranial neuroendoscopic operations. 


\section{Patients and methods}

\section{Patients}

Within 9 years, 121 consecutive patients underwent 126 intracranial endoscopic procedures in combination with neuronavigation; endoscope-assisted procedures were not included. There were 72 men and 49 women; the age ranged between 0.2 and 81 years. In most patients, diagnosis was made by magnetic resonance (MR) imaging. Occlusive hydrocephalus was diagnosed in 57, communicating hydrocephalus in 4, intra- and periventricular tumour in 33, tumour cyst in 3, and CSF-filled cysts (arachnoidal cyst, polycystic hydrocephalus) in 20 patients. In two patients, the cause of the diagnosed hydrocephalus remained unknown. One patient had a post-traumatic abscess in the interhemispheric fissure, and one patient a lobar hematoma.

\section{Neuronavigation}

Before surgery, T1-weighted gradient echo MR images (TE, $4.5 \mathrm{~ms}$; TR, $30 \mathrm{~ms}$; flip angle, 30 ; field of view, $240 \mathrm{~mm}$; scan matrix, $256 \times 256$; slice thickness, $3 \mathrm{~mm}$ ) with external scalp fixed fiducials for image guidance during endoscopy were acquired. This imaging was performed on a 0.5-T or 1.5-T system (Gyroscan ACS NT, Philips Medical Systems, Best, The Netherlands), depending on availability. Immediately before surgery, the MR data were transferred to the work station of the neuronavigational system for reconstruction of an individual three-dimensional model of the patient's head and brain. For neuronavigation, either the EasyGuide Neuro ${ }^{\mathrm{TM}}$ (Philips Medical Systems, Best, the Netherlands) or the Stealth Station $^{\text {TM }}$ (Medtronic SNT, USA) had been used. Both are infrared-based neuronavigational devices, which consist of a mobile workstation, an optical localizing system with two infrared-sensitive cameras and pointers with three lightemitting diodes. The software of the EasyGuide Neuro ${ }^{\mathrm{TM}}$ allowed elongating the pointer virtually for operative path definition, whereas the Stealth Station ${ }^{\mathrm{TM}}$ enabled the surgeon to plan and display the operative path to the target, to which the pointer has to be aligned. Attachment of infrared light-emitting diodes to the rigid endoscope itself is possible with both neuronavigational devices, but was not routinely used.

\section{Surgery}

The operation was done by three neurosurgeons with experience in endoscopy and neuronavigation. All procedures were performed under general anaesthesia with the head rigidly fixed. The coordinates of the reconstructed 3- dimensional model of the patient's head and brain were correlated with the intraoperative head position by touching the skin fiducials on the patients head. Before draping, the burr hole site and the optimum straight trajectory to the target area were selected by virtual pointer elongation (EasyGuide Neuro ${ }^{\mathrm{TM}}$ ) or by path preplanning (Stealth Station $\left.^{\mathrm{TM}}\right)$. After skin incision, burr hole trephination and dura opening, a self-developed articulated arm with a guiding tube for both the neuronavigational pointer and the endoscope was brought in place, adjusted and fixed according to the pre-selected trajectory [11]. The rigid endoscope (Aesculap AG, Tuttlingen, Germany) was introduced into the guiding tube and slowly advanced along the pre-selected trajectory under direct visualization. Only in selected cases with target areas not accessible on a straight trajectory, substantially distorted anatomy or subependymal targets, infrared light-emitting diodes were attached to the endoscope for direct intraoperative navigation of the endoscope tip. At target, ETV, cyst fenestration, biopsy or partial tumour resection was performed according to the standards: In ETV, the floor of the third ventricle was almost exclusively opened with the Fogarty catheter, the opening was enlarged by balloon inflation and the endoscope was introduced through the opening to visualize the basilar artery and rule out further membranes. Cysts were opened with the Fogarty catheter, bipolar coagulation or scissors. Biopsies and partial tumour removal were performed with the grasping forceps with and without prior coagulation.

\section{Evaluation}

After each operation, the surgeon had to define the value of neuronavigation. Three categories were given: (1) not beneficial: the surgical procedure was not influenced by neuronavigation; the neuronavigational system was not used despite its availability; (2) beneficial, but not essential: the surgeon used neuronavigation during surgery, but would have been able to realize the operation without neuronavigational help; tools other than neuronavigation (fluoroscopy, ultrasound) could have provided the surgeon with the same information; 3) essential: the surgeon would not have performed the procedure without neuronavigation; other tools could not compensate for non-availability of neuronavigation. In selected cases, the surgeon reviewed the videotapes before final decision making.

\section{Results}

Overall, in 55 of the 126 intracranial endoscopic procedures (43.7\%), neuronavigation was considered to be not helpful for safe and successful completion of the operation. The 


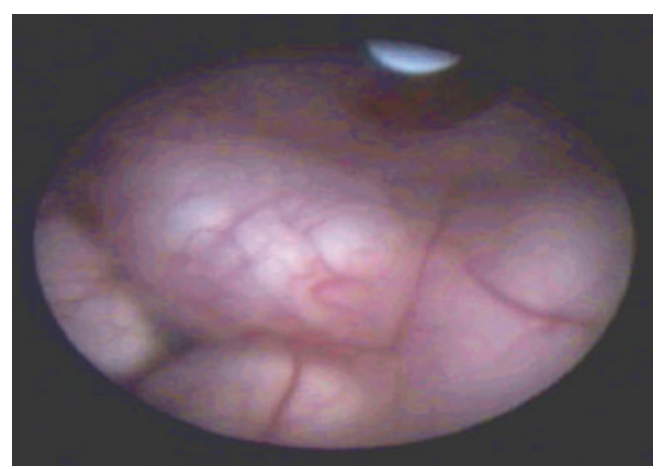

Fig. 1 Endoscopic view of the third ventricular floor in a patient with occlusive hydrocephalus. The mamillary bodies are clearly visible. The bulging, thick and opaque third ventricular floor does not allow the localization of the basilar artery prior to puncture. Neuronavigation was helpful to select a puncture site in a safe distance to the basilar artery

availability of neuronavigation was beneficial in 50 (39.7\%) and essential in $21(16.7 \%)$ procedures. The value of neuronavigation strongly depended on the type of the endoscopic operation.

\section{ETV}

Sixty-five ETVs were performed in 63 hydrocephalic patients. ETV was successful in 46 patients $(70.8 \%$ ) (mean follow-up period of 2.5 years). The mortality and permanent morbidity rate were $0 \%$. In 48 ETVs with normal endoscopic anatomy $(73.9 \%)$, neuronavigation was not beneficial for the surgeon. In 16 ETVs (24.6\%), the surgeon considered neuronavigation to be beneficial for optimizing the burr hole site $(n=3)$, puncture of a small lateral ventricle $(n=3)$, identification of the optimal trajectory through a small foramen of Monro $(n=2)$ and perforation of an opaque, thick tuber cinereum without visualisation of the basilar artery $(n=12)$ (Fig. 1). In one patient with a non-ruptured giant basilar tip aneurysm and occlusive hydrocephalus, the use of neuronavigation was essential for safe perforation of the third ventricular floor (Fig. 2).
Tumour biopsy, tumour resection

Twenty-eight biopsies were performed in patients with intra- and periventricular tumours. Twenty-five of the biopsies were combined with ETV, septostomy or ventriculocystostomy for hydrocephalus therapy or tumour cyst drainage. A diagnosis could be made in 27 of the 28 biopsies (96.4\%). Four tumour resections (one complete resection, three partial resections to open the foramen of Monro) were performed. In four procedures, tumour tissue was not harvested again because of already proven diagnosis. Four endoscopic procedures finally failed and required further operation (three ETV failures, one tumour cyst refilling). We experienced one intratumoural bleeding 9 days after uneventful biopsy, one transient CSF leakage and one transient hemiparesis accounting for a morbidity rate of $8.3 \%$. Neuronavigation was not required in 6 of the 36 operations $(16.7 \%)$. Neuronavigation was beneficial in 19 operations $(52.7 \%)$ to safely perforate the interventricular septum $(n=6)$, to localise the tumour $(n=6)$, to open intratumoural cysts to the ventricular system $(n=3)$, to avoid delicate neural structures and regain orientation during resection $(n=3)$ and to select the best trajectory to the tumour $(n=1)$ (Fig. 3). In 11 procedures (30.6\%), neuronavigation was considered to be essential to localize the lesion and identify a safe biopsy site $(n=5)$, to puncture slit ventricles $(n=2)$, to interconnect ventricles and tumour cysts $(n=3)$ and for orientation in tumour cysts $(n=1)$.

\section{Non-tumourous cystic lesions}

Twenty-three fenestrations of non-tumourous cysts to the basal cistern or the ventricular system were performed in 20 patients. In two patients with post-infectious multicompartmental hydrocephalus and one child with a trapped lateral ventricle after craniosynostosis surgery and CSF shunt placement, the endoscopic therapy finally failed.
Fig. 2 Computerized tomography scan (left) and intraoperative screen display of the navigated endoscope (right) in a 67-year-old female patient with obstructive hydrocephalus due to a giant non-ruptured basilar tip aneurysm. Neuronavigation was essential for opening the third ventricular floor in close vicinity to the basilar tip aneurysm
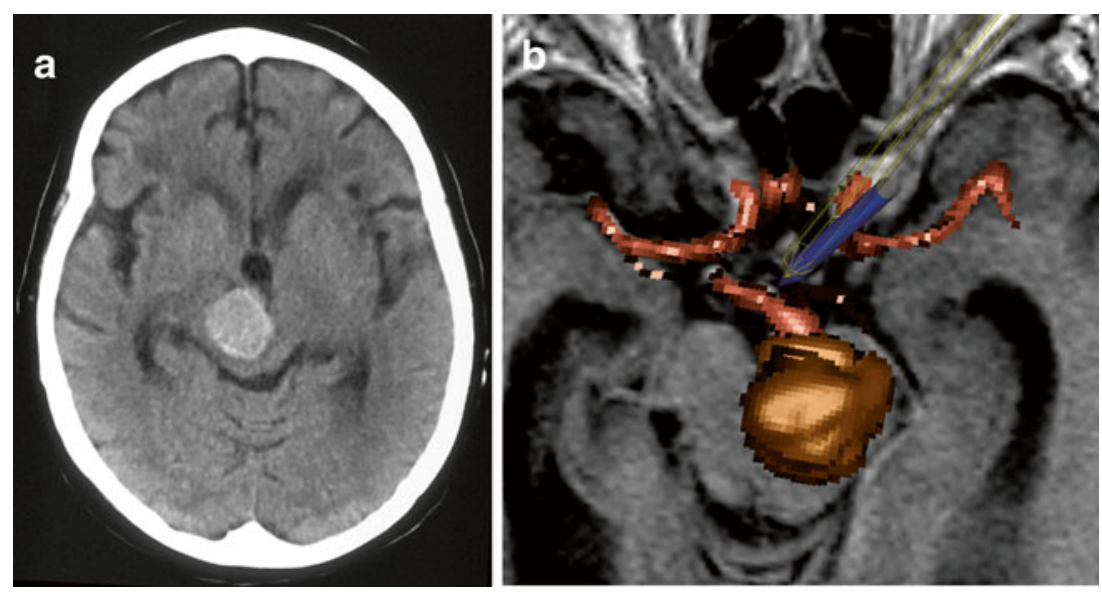

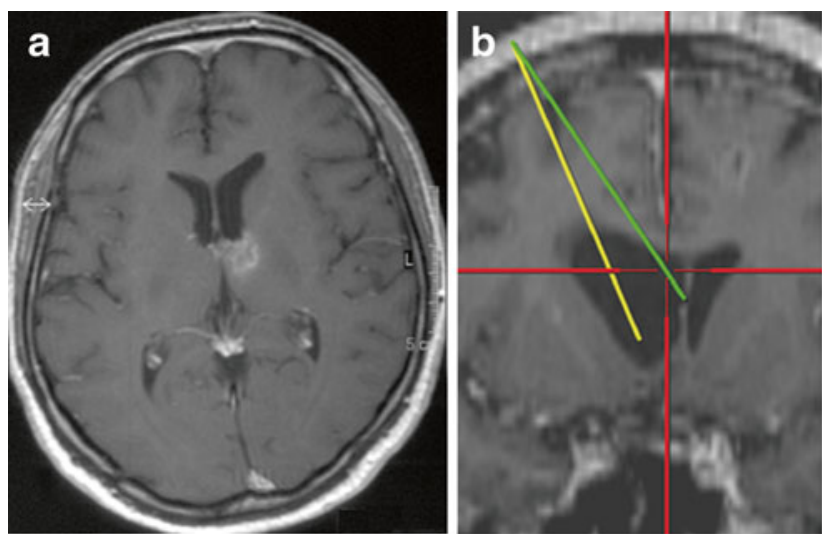

Fig. 3 a Magnetic resonance images of a 45-year-old male with a malignant tumour in the caudate nucleus and a monoventricular hydrocephalus. Tumour biopsy and restoration of the CSF flow by septostomy were the aims of the endoscopic procedure. b

Neuronavigation was not required in one procedure, but was considered to be beneficial in 14 operations $(60.9 \%)$ for intracystic orientation $(n=7)$, definition of the best trajectory $(n=5)$ and a safe stoma site $(n=2)$. In eight procedures (34.8\%), neuronavigation was essential for identification of perforation site and orientation ( $n=4$ each) (Fig. 4).

Abscess, hematoma

One post-traumatic abscess in the interhemispheric fissure was endoscopically aspirated via the lateral ventricle. Neuronavigation was essential to localize the small abscess (Fig. 5). For hematoma removal, neuronavigation was beneficial to insert the endoscope along the main axis of the clot.
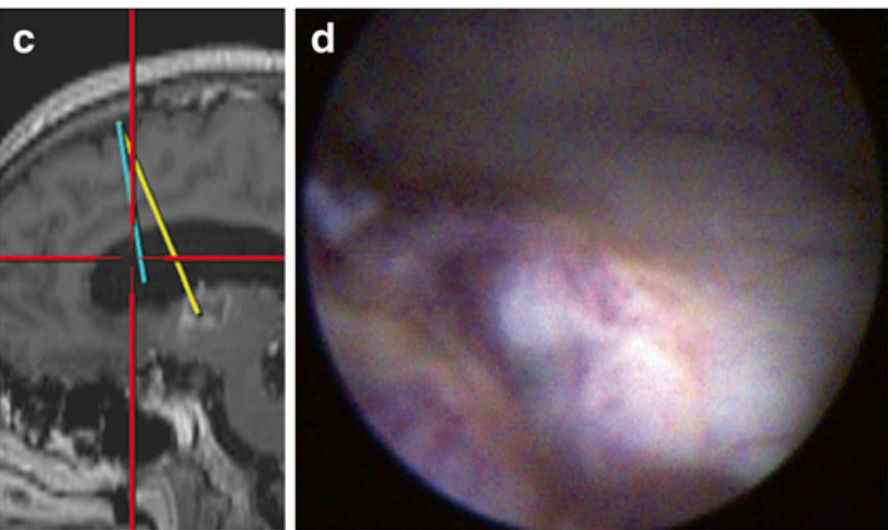

Intraoperative endoscopic view of the biopsy site. Neuronavigationally defined trajectories for septostomy (c) and tumour biopsy (d). Neuronavigation was considered to be beneficial for septostomy

\section{Discussion}

Limitation of the study

Clear criteria to define the usefulness and uselessness of a surgical tool are lacking. Directly after surgery, the surgeon had to categorize the role of navigation. Despite the definition of three categories, the results depend in parts on the subjective impression of the surgeon and, in consequence, could be biased by the growing surgeon's experience and his attitude towards neuronavigational technology. On one hand, one could assume that growing experience is resulting in a decreasing need for neuronavigation. On the other hand, with growing experience, the focus of the surgeon could be more directed towards further optimization of the
Fig. 4 A 70-year-old female with a left temporal AVM which was treated radiosurgically. The patient developed an intraventricular cyst which was progressive in volume and caused a visual field defect. During the endoscopic procedure with neuronavigationally tracked endoscope tip, the small occipital horn was first entered following the predefined yellow trajectory $(l e f t)$, then, on a new trajectory, the cyst was entered (middle) and finally opened to the temporal horn (right). Navigation was essential for ventricle puncture and anatomical orientation
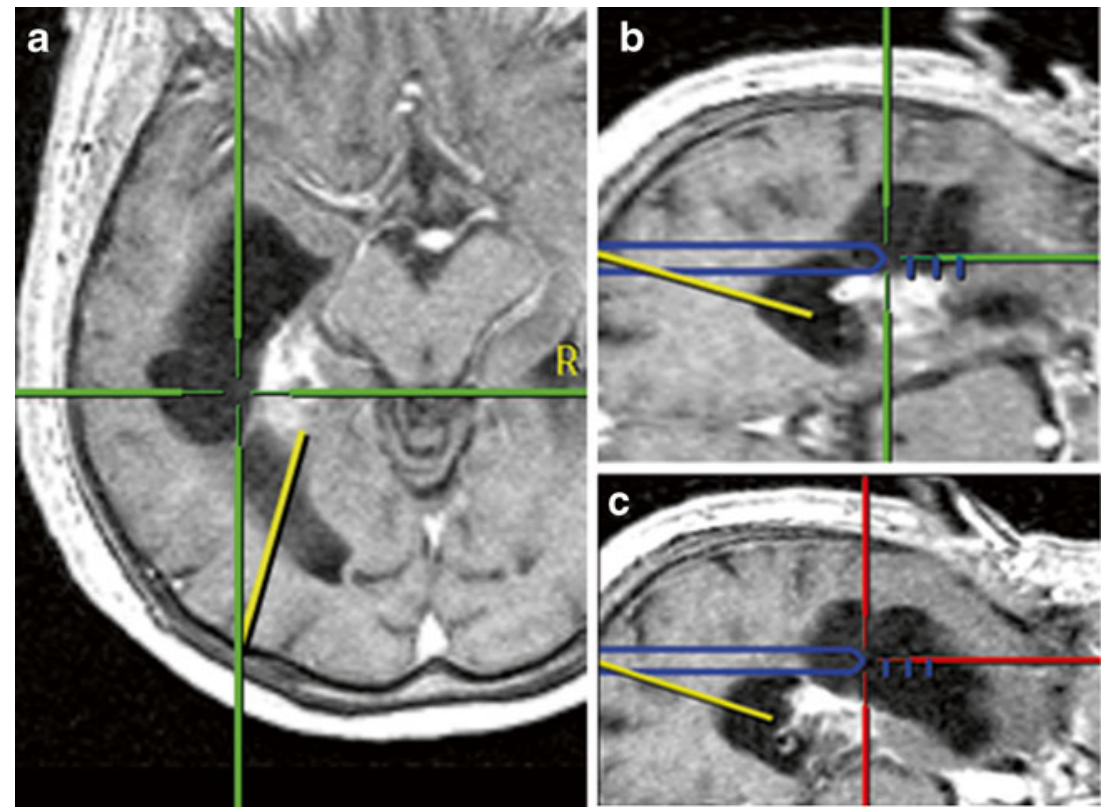

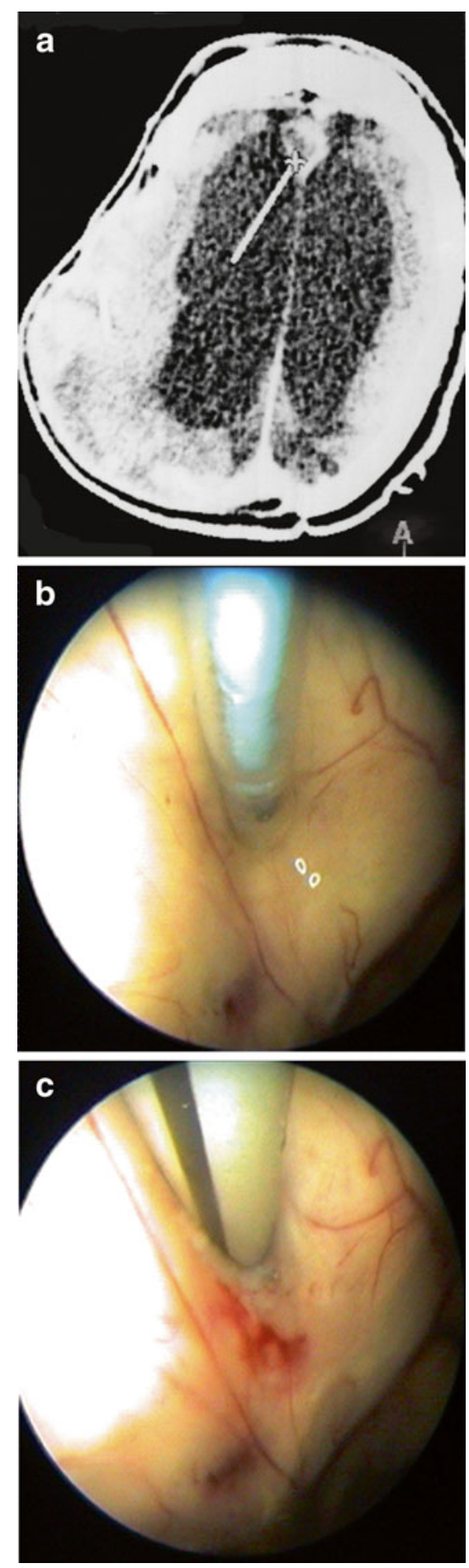

Fig. 5 Computerized tomography scan axial with the neuronavigationally displayed tip of the endoscope (a) in a 25 -year-old patient with a post-traumatic abscess in the interhemispheric fissure. Endoscopically, the anteromedial ventricular wall was perforated (b), and the abscess was aspirated (c). For puncture of the small abscess, neuronavigation was required

procedure resulting in a more frequent use of neuronavigation. However, as only surgeons with endoscopic and neuronavigational expertise prior to the initiation of the prospective study participated, the bias was considered to be minimal.

Neuroendoscopy and navigation

In the recent years, several frameless stereotactic localizing systems (neuronavigation) had been developed and were routinely used [7, 13, 24]. Rhoten and coworkers and our research group were among the first who linked neuronavigation with endoscopy during intracranial operations [12, $16]$. Both hypothesized that safety and efficacy of neuroendoscopic procedures could be enhanced by adding frameless stereotaxy and believed that (1) selection of burr hole site and trajectory before penetrating the cortex and white matter reduces endoscope movements during the procedure, (2) lack of anatomical landmarks as well as poor sight could be compensated by neuronavigation and (3) structures not visible endoscopically could be localized.

The suggestion was made to use routinely neuronavigation during endoscopic operations, but this point of view was not generally shared [18]. The major argument against was that neuronavigation is time consuming, requires additional imaging and is often not necessary in standard endoscopic procedures because of adequate visual control and orientation [22]. It was proposed to limit application of neuronavigation to selected cases (Table 1). This ongoing debate stimulated the initiation of this prospective study. Since 1997, neuronavigation was made routinely available during every intracranial neuroendoscopic operation at the author's institution.

Value of navigation in endoscopic procedures

In the authors' series, neuronavigation was not needed in 48 of 65 ETVs. The hydrocephalic ventricular system allowed safe puncture of the lateral ventricle and early identification of structures leading to the translucent floor of the third ventricle. The basilar artery could be visualized prior to puncture. In the remaining 17 cases, however, neuronavigation was

Table 1 Accepted indications for neuronavigation in neuroendoscopy in the literature

\begin{tabular}{|c|c|c|}
\hline Procedure & Problem & Role of navigation \\
\hline ETV & $\begin{array}{l}\text { Small lateral and third } \\
\text { ventricles }\end{array}$ & Trajectory selection \\
\hline Colloid cyst & Attachment of cyst & $\begin{array}{l}\text { Trajectory selection, } \\
\text { burr hole site }\end{array}$ \\
\hline $\begin{array}{l}\text { Periventricular } \\
\text { tumour }\end{array}$ & $\begin{array}{l}\text { Ependymal covering } \\
\text { of tumour }\end{array}$ & Localizing \\
\hline Pellucidotomy & Slippage of the catheter & $\begin{array}{l}\text { Trajectory + perforation } \\
\text { site selection }\end{array}$ \\
\hline Cystic lesion & $\begin{array}{l}\text { No anatomical landmarks } \\
\text { inside of cyst }\end{array}$ & $\begin{array}{l}\text { Trajectory + perforation } \\
\text { site selection }\end{array}$ \\
\hline
\end{tabular}


useful during ETV. In the majority of these patients, the third ventricular floor was not translucent and did not allow identifying the basilar artery, but neuronavigation allowed selecting a stoma site in safe distance to the vessel. Some authors propose to abandon ETV in cases of an opaque third ventricular floor to avoid damage of the basilar artery [2]. Our results indicate that the neurosurgeon can proceed safely with ETV even in patients with opaque third ventricular floor if neuronavigation is available. In the remaining cases, neuronavigation helped to puncture small lateral ventricles and to advance the endoscope through a small foramen of Monro. Repeated attempts of ventricular puncture and the use of a less suitable trajectory with the risk of fornix damage and endoscope movements in the brain for trajectory correction possibly could be avoided. Preoperative MR imaging allows identification of a small ventricular system and interventricular foramen, but fails to detect thickening and opacity of the third ventricular floor, which are by far the most common variations of the normal endoscopic anatomy [17]. Thus, preoperative MR imaging cannot guide the decision whether or not neuronavigation should accompany endoscopic ventriculostomy for safety and efficacy reasons. The neurosurgeon has to keep in mind that the decision against routine use of neuronavigation in ETV will confront him in more than a fifth of the patients with an intraoperative situation in which the availability of neuronavigation could be desirable.

Neuronavigation was useful in 30 of 36 endoscopic tumour biopsies and resections. Neuronavigation allowed orientation in patients with distorted anatomy due to tumour growth and safe opening of obstructed cerebrospinal fluid pathways either by tumour removal, by ETV or septostomy. Some of the tumours have not been endoscopically visible on the ventricular surface because of subependymal tumour spread. Neuronavigation was essential to select that biopsy site, which allowed harvesting typical tumour tissue. Based on these experiences, the authors recommend using neuronavigation routinely in intra- and periventricular tumour cases.

In all endoscopic fenestrations of non-tumourous cysts either to the ventricular system or to the basal cisterns, neuronavigation was essential for a safe and successful completion of the operation $[6,23,25]$. Especially the loss of anatomical landmarks in large cysts and the need to use different trajectories to perforate one or more cysts explains why frameless stereotaxy was considered to be an indispensable tool. There were no suprasellar and no temporal arachnoidal cysts in the series, which possibly could be treated successfully without neuronavigational help [22].

\section{Brain shift}

Brain shift did not play a major role in our series, making tools for brain shift compensation such as fluoroscopy or intraoperative ultrasound dispensable. In ETV and intra- or periventricular tumours, brain shift was negligible, which is not surprising because experiences in deep brain stimulation already have shown the existent, but minimal, shift of deepseated structures in or close to midline. In endoscopic cyst fenestrations, in which brain shift might play a larger role, brain shift was minimized by patient positioning with the entry point being the upmost point of the head [23].

\section{Conclusion}

In summary, in almost $50 \%$ of intracranial endoscopic procedures, neuronavigation was a helpful adjunct for the surgeon. The application of neuronavigation allowed defining the best trajectory to the target, to proceed with the operation despite the lack of anatomical landmarks, to overcome problems of poor sight and to identify hidden structures such as subependymal tumour tissue or the basilar artery beneath an opaque third ventricular floor. The authors assume that the safety of intracranial neuroendoscopic procedures is enhanced thereby [9]. The proof of this assumption requires the conduction of a prospective randomized trial, which is not likely to come. It can be assumed that the value of neuronavigation for the individual neurosurgeon is not constant over the time; the surgeon's experience, the ease of having neuronavigation available, re-imbursement and changing patient population might be of substantial influence and might result in a more tailored use of neuronavigation. The preoperative MR images likewise might allow to identify patients in which neuronavigation is especially helpful. However, as seen in this study, a substantial percentage of patients exist in which anatomical variants which had not been identified by preoperative MR images rendered neuronavigation useful, leading to the question if a less-tailored use could not be of benefit for the individual patient. In the author's opinion, the optimum management of patients undergoing an intracranial endoscopic procedure includes the use of neuronavigation.

Open Access This article is distributed under the terms of the Creative Commons Attribution Noncommercial License which permits any noncommercial use, distribution, and reproduction in any medium, provided the original author(s) and source are credited.

\section{References}

1. Ellenbogen RG, Moores LE (1997) Endoscopic management of a pineal and suprasellar germinoma with associated hydrocephalus: technical case report. Minim Invas Neurosurg 40:13-16

2. Grant JA, McLone DG (1997) Third ventriculostomy: a review. Surg Neurol 47:210-212 
3. Gumbrecht H, Trost HA, Lumenta CB (2000) Neuroendoscopy combined with frameless neuronavigation. $\mathrm{Br} \mathrm{J}$ Neurosurg 14:129-131

4. Hopf NJ, Grunert P, Fries G, Resch K, Perneczky A (1999) Endoscopic third ventriculostomy: outcome analysis of 100 consecutive procedures. Neurosurgery 44:795-806

5. Jones RFC, Stening WA, Brydon M (1990) Endoscopic third ventriculostomy. Neurosurgery 26:86-92

6. Karabatsou K, Hayhurst C, Buxton N, O'Brien DF, Mallucci CL (2007) Endoscopic management of arachnoid cysts: an advancing technique. J Neurosurg 106:455-462

7. Kikinis R, Gleason PL, Moriarty TM, Moore MR, Alexander E III, Stieg PE, Matsmae M, Lorensen WE, Cline HE, Black PM, Jolesz FA (1996) Computer-assisted interactive three-dimensional planning for neurosurgical procedures. Neurosurgery 38:640 651

8. Leonardo J, Hanel RA, Grand W (2009) Endoscopic tracking of a ventricular catheter for entry into the lateral ventricle: technical note. Minim Invas Neurosurg 52:287-289

9. Longatti P, Fiorindi A, Feletti A, Baratto V (2006) Endoscopic opening of the foramen of Magendie using transaqueductal navigation for the membrane obstruction of the fourth ventricle outlets. Technical note. J Neurosurg 105:924-927

10. McLaughlin MR, Wahlig JB, Kaufmann AM, Albright AL (1997) Traumatic basilar aneurysm after endoscopic third ventriculostomy: case report. Neurosurgery 41:1400-1404

11. Reinges MHT, Spetzger U, Rohde V, Adams L, Gilsbach JM (1998) Experience with a new multifunctional instrument holder in minimally invasive navigated neurosurgery. Minim Invas Neurosurg 41:149-151

12. Rhoten RLP, Luciano MG, Barnett GH (1997) Computer-assisted endoscopy for neurosurgical procedures: technical note. Neurosurgery 40:632-638

13. Roberts DW, Strobehn JW, Hatch JF, Murray W, Kettenberger H (1986) A frameless stereotaxic integration of computerized tomographic imaging and the operating microscope. J Neurosurg 65:545-549

14. Robinson S, Cohen AR (1997) The role of neuroendoscopy in the treatment of pineal region tumours. Surg Neurol 48:360-367

15. Rodziewicz GS, Smith MV, Hodge CJ (2000) Endoscopic colloid cyst surgery. Neurosurgery 46:655-662

16. Rohde V, Reinges MHT, Krombach GA, Gilsbach JM (1998) The combined use of image-guided frameless stereotaxy and neuroendoscopy for the surgical management of occlusive hydrocephalus and intracranial cysts. Br J Neurosurg 12:531-538

17. Rohde V, Gilsbach JM (2000) Anomalies and variants of the endoscopic anatomy for third ventriculostomy. Minim Invas Neurosurg 43:111-117

18. Sangra M, Clark S, Hayhurst C, Mallucci C (2009) Electromagnetic-guided neuroendoscopy in the pediatric population. J Neurosurg Pediatr 3:325-330

19. Schönauer C, Bellotti A, Tessitore E, Parlato C, Moraci A (2000) Traumatic subendymal hematoma during endoscopic third ventriculostomy in a patient with a third ventricle tumour: case report. Minim Invas Neurosurg 43:135-137

20. Schröder HWS, Gaab MR, Niendorf WR (1996) Neuroendoscopic approach to arachnoid cysts. J Neurosurg 85:293-298

21. Schröder HWS, Warzok RW, Assaf JA, Gaab MR (1999) Fatal subarachnoid hemorrhage after endoscopic third ventriculostomy. J Neurosurg 90:153-155

22. Schröder HWS, Wagner W, Tschiltschke W, Gaab MR (2001) Frameless neuronavigation in intracranial endoscopic neurosurgery. J Neurosurg 94:72-79

23. Schulz M, Bohner C, Knaus H, Haberl H, Thomale UW (2010) Navigated endoscopic surgery for multiloculated hydrocephalus in children. J Neurosurg Pediatr 5:434-442
24. Watanabe E, Watanabe T, Manaka S, Mayanagi Y, Takakutra K (1987) Three dimensional digitizer (neuronavigator): new equipement for computed tomography guided stereotaxic surgery. Surg Neurol 27:543-547

25. Weinzierl MR, Coenen VA, Korinth MC, Gilsbach JM, Rohde V (2005) Endoscopic transtentorial ventriculocystostomy and cysto-ventriculo-peritoneal shunt in a neonate with DandyWalker malformation and associated aqueductal obstruction. Pediatr Neurosurg 41:272-277

\section{Comments}

Charlie Teo, Randwick, Australia

The premise of this study was to answer the question of the necessity of neuronavigation for pure endoscopic procedures. The question is pertinent as navigation does increase operative time and cost, and most endoscopists would consider its use superfluous in most cases. The only way to answer the question would be a prospective study of two groups, one in which navigation was used despite the surgeon's indifference and one in which it was not used despite the surgeon's preoperative desire for it. The results would then reflect its application. However, experienced neuro-endoscopists develop an inherent algorithm with which they determine the need for navigation, and it would be unreasonable for a surgeon not to use navigation when his/her experience dictates otherwise. Hence, a study such as this one is probably going to be the best we will get. The authors admit it is flawed by subjective retrospective opinion, and even the study methodology is flawed as the surgeon could choose not to use the navigation even when it was set up to be used from the start. Furthermore, many of the cases identified as "navigation necessary" were probably "navigation unnecessary", but it simply increased surgeon confidence.

This study has unfortunately not answered the question of the necessity of navigation for all pure endoscopic cases. However, it has confirmed that experienced and prudent neuro-endoscopists will and should utilise whatever technology is available to reduce risk and improve outcomes for their patients.

\section{Dattatraya Muzumdar, Mumbai, India}

Rohde et al. present their experience in the role of neuronavigation in the neuroendoscopic procedures. They have reported a prospective study in 121 patients comprising of 126 procedures including endoscopic third ventriculostomy, tumour biopsy/resection, nontumourous and arachnoid cysts as well as hematoma and abscess removal. The aim of this prospective study was to define the benefit of neuronavigation for successful completion of intracranial neuroendoscopic operations. They suggest integration of neuronavigation into the routine neuroendoscopic tumour operations.

The role of use of neuronavigation during neuroendoscopic procedures is controversial and can be debated. Although the issue has been described in the literature, there is no clear consensus in the definition of neuronavigation use in neuroendoscopic procedures. The manuscript is well written and discussed. The study is prospective. The sample size is reasonable although a larger sample size or a multicentric study would have been more effective. The parameters used for evaluation of the results, i.e. benefical or essential, are not robust and largely subjective.

The limitations of this study are more crucial, and the authors have discussed it, namely the subjective bias of the operating surgeon. A randomized controlled study would have been ideal but is difficult to design. The authors mention that only surgeons with endoscopic and neuronavigational expertise were included in the prospective study to minimize the bias. The inability to effectively use neuronavigation 
following CSF drainage due to volume shift is known. In such a situation, intraoperative ultrasound and fluoroscopy can be helpful.

Neuronavigation is a useful adjunct during neuroendoscopic procedures, and its application in selected cases could enhance the safety of the neuroendosopic procedure, thus ensuring a gratifying result and outcome. A significant personal bias could ensue in the initial experience of the surgeon performing the procedure. A tailored judgement in the use of neuronavigation during neuroendoscopic procedures would follow with increasing experience in this regard over a period of time.

A large multicentric study across the world including prominent centres performing neuroendoscopic procedures should relate their experience. This will certainly help to define guidelines for use of neuronavigation during neuroendoscopic procedures. It will also help provide a clear message to the young enthusiastic surgeons interested in neuroendoscopy 